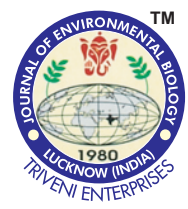

\title{
Adsorption of nickel ions by surface modified magnetite nanoparticles: Kinetics study
}

Authors Info
Carlin Geor Malar',
M. Seenuvasan',3* and
K. Sathishkumar',
'Department of Biotechnology,
Rajalakshmi Engineering College,
Chennai-602 105, India
${ }^{2}$ Department of Petrochemical
Engineering, SVS College of
Engineering, Coimbatore-642 109,
India
${ }^{3}$ Department of Chemical
Engineering, Hindusthan College
of Engineering and Technology,
Coimbatore-641 032, India
${ }^{4}$ Department of Chemical
Engineering, SSN College of
Engineering, Chennai-603 110,
India
Revited by
Professor G. Manjula
Dr. M. Anil Kumar
Dr. S. Subhagar
*Corresponding Author Email :
msvasan.chem@gmail.com

\begin{abstract}
Aim: In this study, the adsorption ability of surface modified magnetite nanoparticles was investigated.

Methodology: Magnetite nanoparticles (MNs) were prepared by solvothermal method and the surface was modified by 3-(2-Aminoethylamino) propyldimethoxymethylsilane (AEAPS) and utilized as an adsorbent against nickel ions in aqueous solution. Characteristic studies were performed to study the various properties of MNs before and after surface modification. $\mathrm{Ni}_{2}{ }^{+}$adsorption was studied as a function of contact time, initial concentration and adsorbent dosage at neutral $\mathrm{pH}$ and constant temperature $\left(100^{\circ} \mathrm{C}\right)$.
\end{abstract}

Results: Presence of required characteristic peaks in IR spectrum and X-ray diffraction (XRD) patterns revealed formation of magnetite and surface modification. Spherical and defined morphology of surface modified MNs (aMNs) was studied through electron micrographs. The kinetic data obeyed the first-order equation.

Interpretation: Surface modified MNs can be used for the efficient removal of $\mathrm{Ni}_{2}{ }^{+}$from aqueous solution. Also, the kinetics study revealed the adsorption capacity of MNs against nickel ions.

Key words: Adsorption, Kinetics, Magnetite, Nanoparticles, Nickel ions

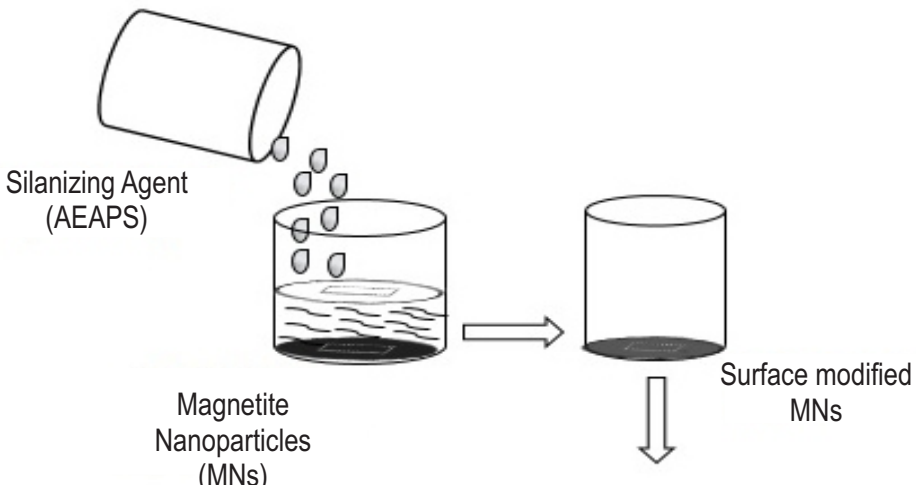

(MNs)
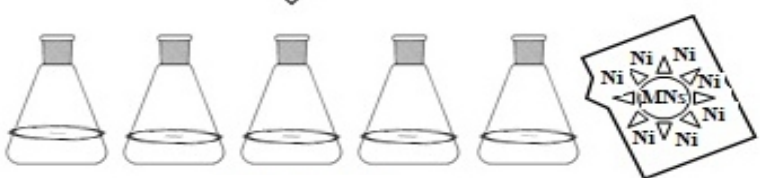

Nickel ion

Adsorption

How to cite : Malar, Carlin Geor, M. Seenuvasan, K. Sathishkumar: Adsorption of nickel ions by surface modified magnetite nanoparticles: Kinetics study. J. Environ. Biol., 40, 748-752 (2019). DOI : http://doi.org/10.22438/jeb/40/4(SI)/JEB_10 


\section{Introduction}

Release of organic and inorganic compounds into the environment tremendously pollutes the aquatic ecosystem. Effluents released by the human activities turn toxic over prolonged accumulation (El-Shahawi et al., 2010; Andreu et al., 2016; Li et al., 2015; Luna-Acosta et al., 2015). Metallic compounds used for various industrial processes are carried as trace elements in the waste water.

It becomes important to remove these toxic heavy metals from the waste water by an efficient method. Recently, nanoparticles are effectively involved in the heavy metal adsorption because of their higher surface area property (Seenuvasan et al., 2013). Amongst various nanoparticles, magnetite (MNs) outstand in their versatile physical and chemical properties (Seenuvasan et al., 2014; Keyhanian et al., 2016, Balaji et al., 2016) that makes them suitable to be used as an adsorbent. Also, uniformly sized monodisperse MNs serve as an excellent adsorbent are widely synthesized by simple solvothermal method (Yanez-vilar et al., 2009; Sahoo et al., 2012; Zhang et al., 2013; Wang et al., 2013) due to its additional benefits of hydrothermal synthesis (Chaisri et al., 2015; Dar et al., 2015).

The objective of the study was to assess the suitability of surface modified MNs for effective elimination of $\mathrm{Ni}^{2+}$ from aqueous solution. Solvothermally synthesized MNs were modified using AEAPS and the properties were studied using various characterization analysis. Then, the adsorption of nickel on the surface modified MNs was studied as a function of contact time, initial concentration and adsorbent dosage.

\section{Materials and Methods}

Magnetite nanoparticles was synthesized by following a modified solvothermal method (Li et al., 2016). Briefly, $18.5 \mathrm{mM}$ of $\mathrm{FeCl}_{3} \cdot 6 \mathrm{H}_{2} \mathrm{O}$ and $3 \mathrm{mM}$ of sodium acetate was mixed with ethylene glycol and ethylene diamine to form a homogeneous mixture. It was then kept at $200^{\circ} \mathrm{C}$ for $8 \mathrm{hr}$ in a Teflon lined autoclave and the formed dark colored MNs were decanted magnetically and vacuum dried at $60^{\circ} \mathrm{C}$.

Typical surface modification of MNs involved treatment with the silanizing agent (Deng et al., 2013). AEAPS (3 mM) in toluene/methanol mixture $(1: 1 \mathrm{v} / \mathrm{v})$ was added to the MNs suspension and kept at $80^{\circ} \mathrm{C}$ for $6 \mathrm{hr}$ under vigorous stirring. The surface modified MNs (aMNs) were decanted magnetically and stored for future use.

Characterization of MNs and aMNs was performed using FT-IR (Perkin Elmer FTIRC 100566) in 4000-400 $\mathrm{cm}^{-1}$ range, Xray diffractometer (XRD, X'Pert3 Powder X-ray Diffractometer) with $\mathrm{Cu}$ Ka radiation at $30 \mathrm{kV}$ and $30 \mathrm{~mA}$, FESEM (FEI quanta FEG 200, Operating voltage $30 \mathrm{kV}$ ) and BET (Quantachrome ASiQwin) analysis.
Adsorption was performed as batch experiments by mixing $10 \mathrm{ml}$ nanoparticles suspension and $10 \mathrm{ml}$ of nickel solution of varying concentration (10 to $50 \mathrm{mg} \mathrm{l}^{-1}$ ). The reaction was performed in shaker with continuous mixing at $100 \mathrm{rpm}$ for 24 $\mathrm{hr}$ (room temperature). Nanoparticles were than removed by centrifugation and the nickel left in the supernatant was measured in Atomic Absorption Spectroscopy (AAS). The nickel concentration in the solution was then calculated by the following equation:

$$
C=\frac{\left(C_{i}-C_{e}\right) V}{m}
$$

Where, $\mathrm{C}, \mathrm{C}_{\mathrm{i}}$ and $\mathrm{C}_{\mathrm{e}}$ are $\mathrm{Ni}^{2+}$ concentration adsorbed on a MNs $\left(\mathrm{mg} \mathrm{g}^{-1}\right)$, initial and equilibrium $\left(\mathrm{mg} \mathrm{l}^{-1}\right)$, respectively; $V$ is the volume (I) and $\mathrm{m}$ is the mass $(\mathrm{mg})$ of the adsorbent.

Many possible models were investigated to understand the kinetics mechanism involved in the $\mathrm{Ni}^{2+}$ adsorption. Firstorder, pseudo-first-order and pseudo-second-order models. On the basis of the experimental data and model, the correlation coefficient $\left(R^{2}\right)$ exhibited the predicted values. Higher $R^{2}$ values were chosen and that model was known to describe the kinetics successfully. Equation (2) gives the first-order rate kinetics.

$$
\log C_{e}=\frac{k_{1}}{2.303} t+\log C_{0}
$$

Where, $\mathrm{C}_{0}$ and $\mathrm{C}_{\mathrm{e}}$ are $\mathrm{Ni}_{2}^{+}$concentration at initial and at time $\mathrm{t}\left(\mathrm{mg} \mathrm{l}^{-1}\right)$ respectively, and $\mathrm{k}_{1}$ is the first-order rate constant $\left(\min ^{-1}\right)$.

Pseudo-first-order kinetics in linear form can be expressed as:

$$
\log \left(q_{0}-q_{e}\right)=\log \left(q_{0}\right)-\frac{k_{1}}{2.303} t
$$

Where, $\mathrm{q}_{\mathrm{o}}$ and $\mathrm{q}_{\mathrm{e}}$ are $\mathrm{Ni}_{2}{ }^{+}$concentration at time 't' and at equilibrium ( $\mathrm{mg} \mathrm{g}^{-1}$ ) and $\mathrm{k}_{1}$ is the rate constant for pseudo-firstorder kinetics $\left(\min ^{-1}\right)$.

Pseudo-second-order kinetics can be expressed as

$$
\frac{t}{q_{e}}=\frac{k_{1}}{k_{2} q_{0}^{2}}+\frac{t}{q_{0}}
$$

Where $\mathrm{k}_{2}$ is the rate constant for second-order kinetics ( $g$ mg $\min ^{-1}$ ) (Kumar et al., 2009)

\section{Results and Discussion}

Fig. 1 depicts the FTIR spectrum of MNs and aMNs. The characteristic peak present at $570 \mathrm{~cm}^{-1}$ corresponds stretching Fe-O vibrations (Seenuvasan et al., 2013a). Bands around 3200 $\mathrm{cm}^{-1}$ and at $1624 \mathrm{~cm}^{-1}$ was due to the presence of hydroxyl groups on the surface of MNs (Seenuvasan et al., 2013a) and the surface modification was observed by peak shift of Fe-O to 556 $\mathrm{cm}^{-1}$. Amino group from AEAPS was evident from the peak at 3112 $\mathrm{cm}^{-1}$ in aMNs (Malar et al., 2018). The FTIR peaks confirmed the formation of MNs and the surface modification of MNS by AEAPS. 


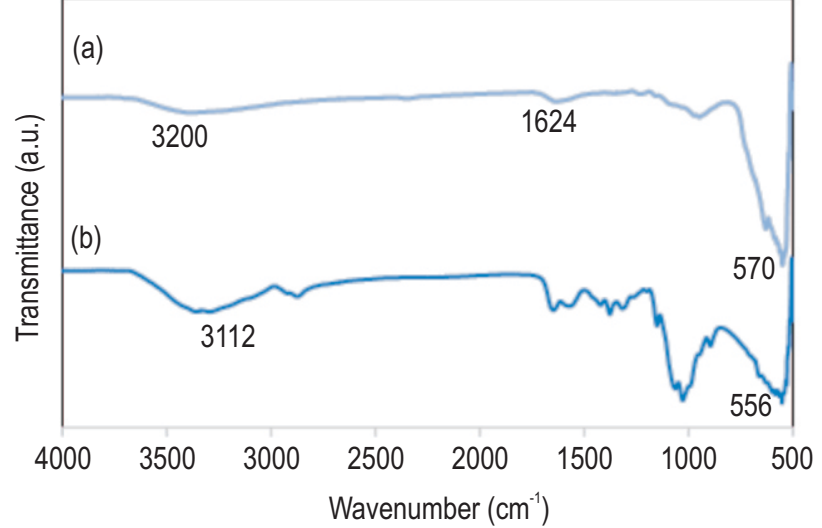

Fig.1 : FT-IR Spectrum of (a) MNs (b) aMNs.

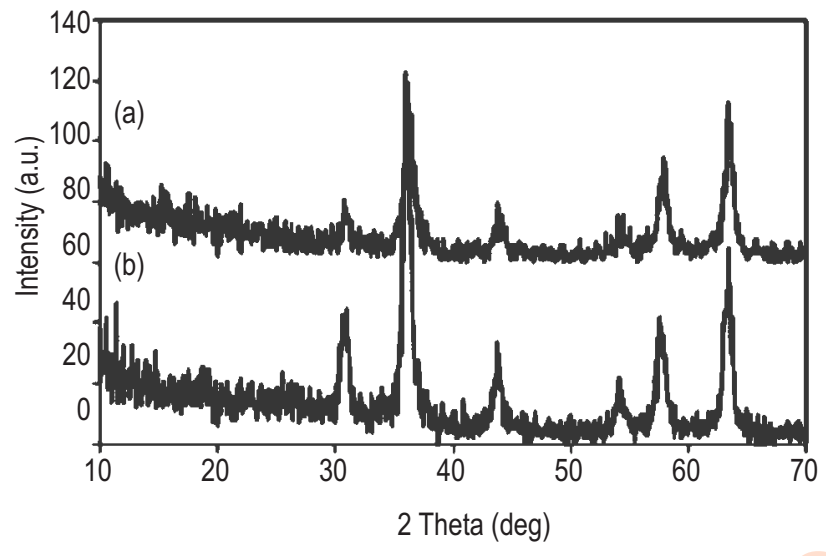

Fig. 2 : XRD pattern of (a) MNs and (b) aMNs.

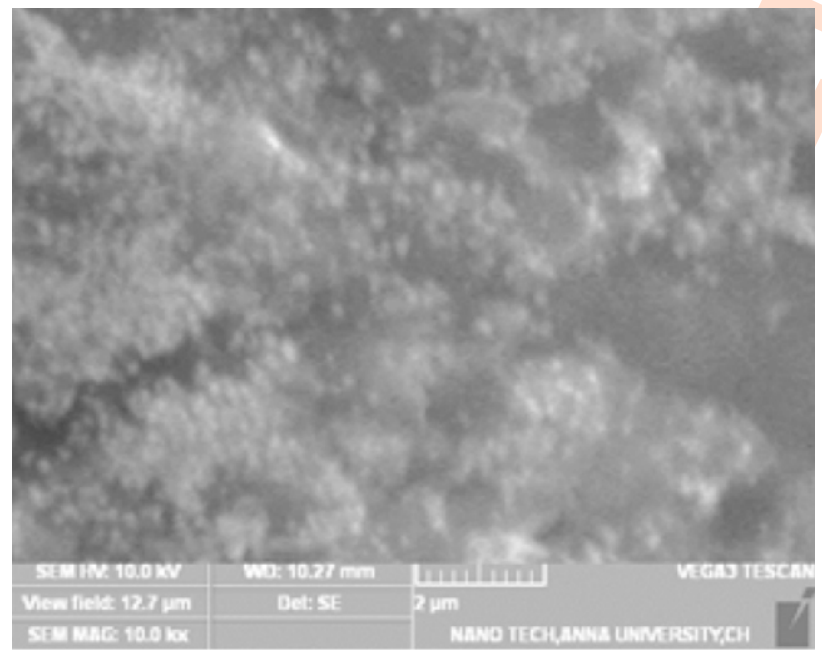

Fig. 3 : FESEM micrograph of aMNs.

Diffraction patterns (Fig. 2) of MNs and aMNs show the characteristic peaks at $31.2^{\circ}, 35.5^{\circ}, 43.1^{\circ}, 57^{\circ}$ and $62.9^{\circ}$ with indices (2 20 ), (3 11 1), (4 00 0), (5 11 1) and (4 4 1) to reveal the inverse spinel structure. Smaller sized crystalline structured MNs are obvious from the sharp broadened peaks. With the help of
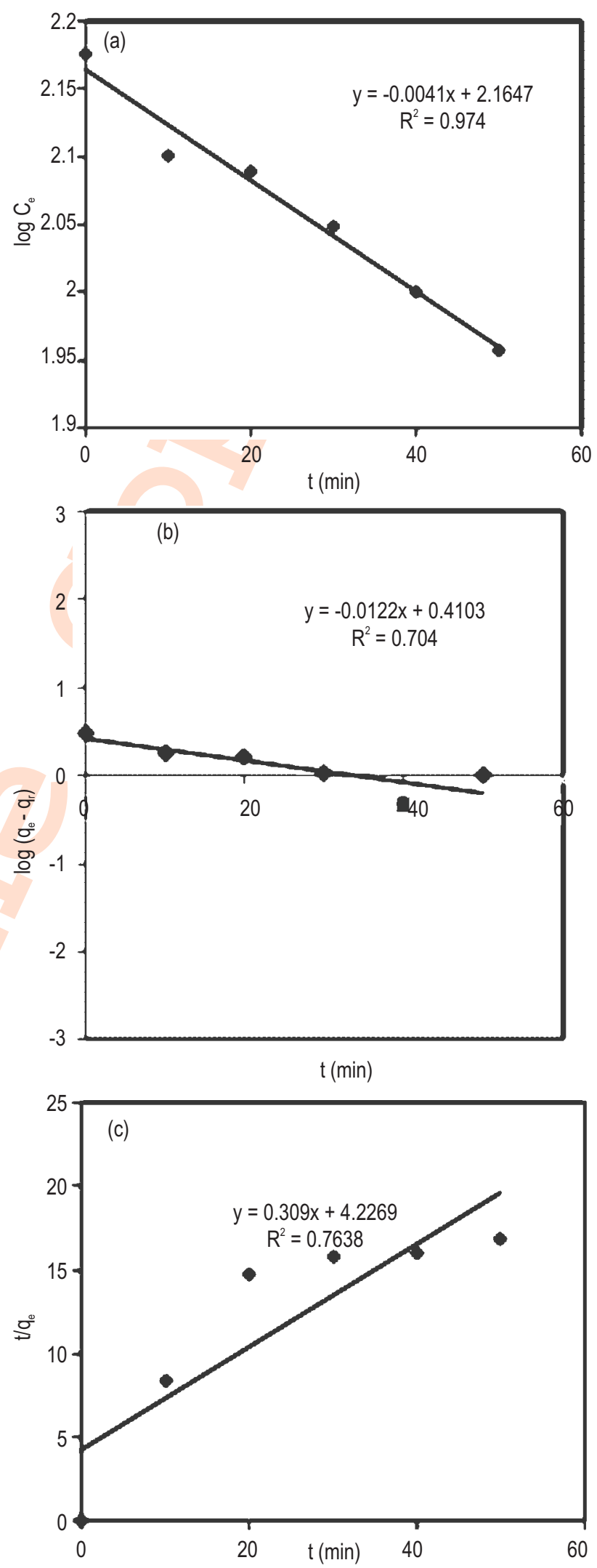

Fig. 4 : Kinetic models (a) first-order, (b) pseudo-first-order and (c) pseudo-second-order and their parameters. 
Table 1 : Kinetic models and their parameters

\begin{tabular}{lll}
\hline Kinetics Model & \multicolumn{2}{c}{ Parameters } \\
\hline First-order equation & $\mathrm{R}^{2}$ & 0.974 \\
Pseudo-first-order equation & $\mathrm{k}_{1}$ & 0.0094 \\
& $\mathrm{R}^{2}$ & 0.704 \\
Pseudo-second-order equation & $\mathrm{k}_{1}$ & 0.0281 \\
& $\mathrm{R}^{2}$ & 0.7636 \\
& $\mathrm{k}_{2}$ & 0.0226 \\
\hline
\end{tabular}

Debye-Scherrer equation $(d=0.9 \lambda / \beta \cos \theta ; \beta$ is the full width at half maximum) on the intense peak, the size was calculated to be $11.53 \mathrm{~nm}$ and $12.22 \mathrm{~nm}$ for MNs and aMNs, respectively.

Irregular and defined spherical morphological appearance was observed from the electron micrographs (Fig. 3) of MNs and aMNs respectively. It was very clear that the surface modification of MNs resulted in uniform distribution of amino groups.

High surface area and porous structure of MNs ease the adsorption capacity. The sorption activity determines the physicochemical properties of the adsorbent. The specific surface areas were found to be $94.32 \mathrm{~m}^{2} \mathrm{~g}^{-1}$ and $93.15 \mathrm{~m}^{2} \mathrm{~g}^{-1}$ for MNs and aMNs, respectively. Reduction in the surface area by surface modification did not affect the adsorption capacity at a notable extent.

For constant initial concentration of metal ions, the rate constant and correlation coefficient were determined using the plot $\log C_{e}$ Vs $t$ (Fig. 4a) that showed a linear fit with highest $R^{2}$ value (0.974). Hence, first-order model can be considered as best fit to describe the adsorption kinetics and the $\mathrm{Ni}^{2+}$ adsorption follows first-order kinetics (Seenuvasan et al., 2018).

Fig. 4c illustrates the plot of $t / q_{e}$ Vs that explains the pseudo-second-order kinetics. Pseudo-second-order kinetics failed to explain the adsorption mechanism due to the deviation. Table 1 gives the comparison of kinetic parameters of all the kinetics models.

The study revealed that surface modified MNs can be an effective adsorbent for the removal of $\mathrm{Ni}^{2+}$ from aqueous solutions. The formation of MNs and surface modification were confirmed by the presence of all the characteristic peaks in FTIR spectrum. Also, nanosized crystalline structure and mesoporous morphology were clear from the XRD patterns and FESEM images respectively. With an insight knowledge on the properties of the aMNs, the adsorption mechanism of $\mathrm{Ni}^{2+}$ from the aqueous solutions were described by the kinetics study. Adsorption of $\mathrm{Ni}^{2+}$ obeys the first-order equation with good relation.

\section{Acknowledgments}

The authors extend their gratitude to the management of SVS College of Engineering, Coimbatore, Department of
Biotechnology, Rajalakshmi Engineering College, Thandalam and Department of Chemical Engineering, SSN College of Engineering, Kalavakkam for continuous support of the study.

\section{References}

Andreu, V., E.G. Garcia., J.A. Pascual., P.V. Roig and Y. Pico: Presence of pharmaceuticals and heavy metals in the waters of a Mediterranean coastal wetland: potential interactions and the influence of the environment. Sci. Total Environ., 540, 278-286 (2016).

Balaji, N., K.S. Kumar, M. Seenuvasan, G. Vinodhini and M.A. Kumar: Immobilization of laccase onto micro-emulsified magnetic nanoparticles for enhanced degradation of a textile recalcitrant. $J$. Environ. Biol., 37,1489-1496 (2016).

Bansal, R.C. and M. Goyal: Activated Carbon Adsorption. CRC Press, New York, 346 (2005).

Chaisri, T., K. Suwannahong, J. Kruenate and T. Kreetachat: Removal of VOCs by photocatalytic oxidation using nano-TiO/PLA biocomposite. J. Environ. Biol., 36, 617-621 (2015).

Dar, M.A., J.A. Wani, S.K. Raina, M.Y. Bhat and M.A. Malik: Relationship of leaf nutrient content with fruit yield and quality of pear. $J$. Environ. Biol., 36, 649-553 (2015).

Deng, J., He, J., Zheng, J.S., Terakawa, S., Huang, H., Fang, F.C., Li, Y., Cheng, P., and L. Jiang: Preparation and application of amino- and dextran-modified superparamagnetic iron oxide nanoparticles. Part. Sci. Technol. 31, 241-247 (2013).

Keyhanian, F., S. Shariati, M. Faraji and M. Hesabi: Magnetite nanoparticles with surface modification for removal of methyl violet from aqueous solutions. Arab. J. Chem., 9, 348-354 (2016).

Kumar, P.S. and K. Kirthika: Equilibrium and kinetic study of adsorption of nickel from aqueous solution onto bael tree leaf powder. J. Eng. Sci. Tech., 4, 351-363 (2009).

Li, X., Y. Yang., X. Xu, C. Xu and J. Hong: Air pollution from polycyclic aromatic hydrocarbons generated by human activities and their health effects in China. J. Clean. Prod., 112, 1360-1367 (2016).

Li, M., Q. Gao, T. Wang, Y.S. Gong, B. Han, K.S. Xia and C.G. Zhou: Solvothermal synthesis of $\mathrm{Mn}_{x} \mathrm{Fe}_{3-x} \mathrm{O}_{4}$ nanoparticles with interesting physico-chemical characteristics and good catalytic degradation activity. Mater. Des., 97, 341-348 (2016).

Luna-Acosta, A., H. Budzinski., K.L. Menach., H.T. Guyon and P. Bustamante: Persistent organic pollutants in a marine bivalve on the Marennes-Oléron Bay and the Gironde Estuary (French Atlantic Coast) part 1: Bioaccumulation. Sci. Total. Environ., 514, 500-510 (2015).

Malar, C.G., M. Seenuvasan and K.S. Kumar: Prominent study on surface properties and diffusion coefficient of urease-conjugated magnetite nanoparticles. Appl. Biochem. Biotechnol., 186, 174185 (2018).

Sahoo, B., S.K. Sahu, S. Nayak, D. Dhara and P. Pramanik: Fabrication of magnetic mesoporous manganese ferrite nanocomposites as efficient catalyst for degradation of dye pollutants. Catal. Sci. Technol., 2, 1367-1374 (2012).

Seenuvasan, M., C.G. Malar, S. Preethi, N. Balaji, J. lyyappan, M.A. Kumar and K.S. Kumar: Fabrication, characterization and application of pectin degrading $\mathrm{Fe}_{3} \mathrm{O}_{4}-\mathrm{SiO}_{2}$ nanobiocatalyst, Mater. Sci. Eng. CMater. Biol. Appl., 33, 2273-2279 (2013 a).

Seenuvasan, M., C.G. Malar, S. Preethi, N. Balaji, J. Iyyappan, M.A. Kumar and K.S. Kumar: Immobilization of pectinase on co- 
precipitated magnetic nanoparticles for enhanced stability and activity. Res. J. Biotech., 8, 24-30 (2013 b).

Seenuvasan, M., J.R.G. Suganthi, G. Sarojini, G.C.G. Malar, M.E. Priya, and M.A. Kumar: Effective utilization of crustacean shells for preparing chitosan composite beads: applications in ameliorating the biosorption of an endocrine disrupting heavy metal. Desal. Wat. Treat., 121, 28-35(2018).

Seenuvasan, M., K.S. Kumar, C.G. Malar, S. Preethi, M.A. Kumar and N. Balaji: Characterization, Analysis, and Application of Fabricated $\mathrm{Fe}_{3} \mathrm{O}_{4}$-Chitosan-Pectinase Nanobiocatalyst. Appl. Biochem. Biotechnol., 172, 2706-2719 (2014).

Wang, T., L.Y. Zhang, H.Y. Wang, W.C. Yang, Y.C. Fu, W.L. Zhou, W.T. Yu,
K.S. Xiang, Z. Sun, S. Dai and L.Y. Chai: Controllable synthesis of hierarchical porous $\mathrm{Fe}_{3} \mathrm{O}_{4}$ particles mediated by poly(diallyldimethylammonium chloride) and their application in arsenic removal. ACSAppl. Mater. Interfaces., 5, 12449-12459 (2013). Yanez-Vilar, S., M. Sanchez-Andujar, C. Gomez-Aguirre, J. Mira, M.A. Sennaris-Rodríguez and S. Castro-Garcia: A simple solvothermal synthesis of MFe2O4 (M=Mn, Co and Ni) nanoparticles. J. Solid State Chem., 182, 2585-2690 (2009).

Zhang, Z.L., Y.H. Wang, Q.Q Tan, Z.Y. Zhong and F.B. Su: Facile solvothermal synthesis of mesoporous manganese ferrite $\left(\mathrm{MnFe}_{2} \mathrm{O}_{4}\right)$ microspheres as anode materials for lithium-ion batteries. J. Colloid Interface Sci., 398, 185-192 (2013). 